\title{
APOE related alterations in cerebral activation even at college age
}

\author{
N Scarmeas, C G Habeck, J Hilton, K E Anderson, J Flynn, A Park, Y Stern
}

J Neurol Neurosurg Psychiatry 2005;76:1440-1444. doi: 10.1136/jnnp.2004.053645

\begin{abstract}
Background: Associations between the APOE genotype and various medical conditions have been documented at a very young age. The association between the APOE genotype and cognitive performance varies at different ages. APOE related changes in brain activation have been recently reported for middle aged and elderly subjects.

Objective: To explore APOE related alterations during cognitive activation in a population of young adults.

Methods: Using $\mathrm{H}_{2}{ }^{15} \mathrm{O}$ positron emission tomography (PET), imaging was carried out in 20 healthy young adults (age 19 to 28 years; four $\epsilon 4$ carriers and 16 non- $\epsilon 4$ carriers) during a non-verbal memory task. Voxel-wise multiple regression analyses were undertaken, with the activation difference PET counts as the dependent variable and the APOE genotype as the independent variable.

Results: Brain regions were identified where $\epsilon 4$ carriers showed significantly lower or higher activation than noncarriers.

Conclusions: The results suggest that APOE dependent modulation of cerebral flow may be present even at a young age. This may reflect an APOE related physiological heterogeneity which may or may not predispose to brain disease in the ensuing decades or, less likely, the effect of very early Alzheimer's disease related pathological changes.
\end{abstract}

$\mathrm{E}$ fects of the APOE genotype on lipid metabolism, ${ }^{1}$ blood pressure, ${ }^{2}$ atherosclerosis, ${ }^{3}$ ischaemic heart disease, myocardial infarction, ${ }^{5}$ and cognitive performance in type I diabetes ${ }^{6}$ have been documented in very young subjects or even in children. ${ }^{78}$ Animal studies have indicated that the APOE genotype seems to affect the stress response and spatial memory ${ }^{9}$ and to regulate synaptic plasticity and long term potentiation in the hippocampus ${ }^{10}$ of young mice. It is therefore conceivable that APOE related alterations in cerebral physiology may exist even from a very young age. Few brain imaging studies have investigated the association between APOE and patterns of brain activation, and all have been carried out in middle aged and elderly individuals who were either cognitively intact ${ }^{1-14}$ or had Alzheimer's disease. ${ }^{15}$ Our group ${ }^{16}$ and others ${ }^{17}$ have recently demonstrated APOE related changes in cerebral flow and metabolism during rest in very young subjects. We now report APOE related alterations during cognitive activation in the same population.

\section{METHODS}

College age right handed healthy young adults (four $\in 4$ carriers and 16 non- $\epsilon 4$ carriers, reflecting the proportion of APOE polymorphism in our population) underwent resting $\mathrm{H}_{2}{ }^{15} \mathrm{O}$ positron emission tomography (PET). Subjects were screened for the absence of any neurological or psychiatric disease by structured clinical interviews, neuropsychological testing, and brain magnetic resonance imaging (MRI).

The non-verbal episodic memory task used in the neuroimaging component of this study (described in more detail elsewhere ${ }^{18}$ ) comprised a simple demand (SD) condition and a titrated demand (TD) condition (fig l). For each scanning session (which included both the encoding and the recognition phases), a bolus of $30 \mathrm{mCi}_{2}{ }^{15} \mathrm{O}$ was injected intravenously. After measured attenuation correction (15 minute transmission scan) and reconstruction by filtered back projection, image resolution was $4.6 \mathrm{~mm}$ full width at half maximum (FWHM). Non-quantitative counts (referred to as relative cerebral blood flow ( $\mathrm{rCBF}$ ) values) were obtained. Eighteen trials for the SD condition and two trials for the TD condition were acquired during each scan. Subjects viewed the shape stimuli on an overhead monochrome monitor while lying in a supine position.

The SPM99 program (statistical parametric mapping) was used to implement realignment, spatial transformation, smoothing (isotropic, Gaussian kernel $(F W H M=12 \mathrm{~mm})$ ), and proportional scaling by global mean. Voxel-wise multiple regression analyses were carried out with $\mathrm{rCBF}$ as the dependent variable. Independent variables were: the cognitive task condition (TD $v$ SD); the APOE genotype (dichotomous form, presence $v$ absence of an $\epsilon 4$ allele); and the cognitive task condition $\times$ APOE interaction. Our primary interest was the statistical significance of the condition $\times$ APOE interaction effect, which relates to the hypothesis that cerebral activation differs between $\epsilon 4$ allele carriers and non-carriers. Cluster sizes of $\geqslant 10$ voxels are reported. We also report the cognitive task condition effect (the difference between TD and SD rCBF). This is meant to represent the cerebral activation related to titrated memory performance, while subtracting activity related to basic sensory and motor processing. The false positive rate was controlled at $\alpha=0.05$ per map (Bonferroni corrected for multiple comparisons).

We tested our data for various assumptions of parametric statistics (PP and QQ plots, Levene's test for equality of variances-homoscedasticity, the Kolmogorov-Smirnov test for normality) and we detected no deviations from the required assumptions. However, given the small sample size it is always possible that some of the above tests may fail to reject the null because of low power and parametric test assumptions may be still violated. Thus to allow for possible non-normality in the distribution of the activation mean of the $\in 4$ carriers, we conducted a random permutation test (which in contrast to parametric tests, does not require any distributional assumptions) at the voxels where the parametric SPM analysis produced significant results.

Abbreviations: $A P O E$, apolipoprotein $E_{;} F W H M$, full width at half maximum; rCBF, relative cerebral blood flow; $S D$, simple demand; SPM, statistical parametric mapping; TD, titrated demand 
Simple demand condition

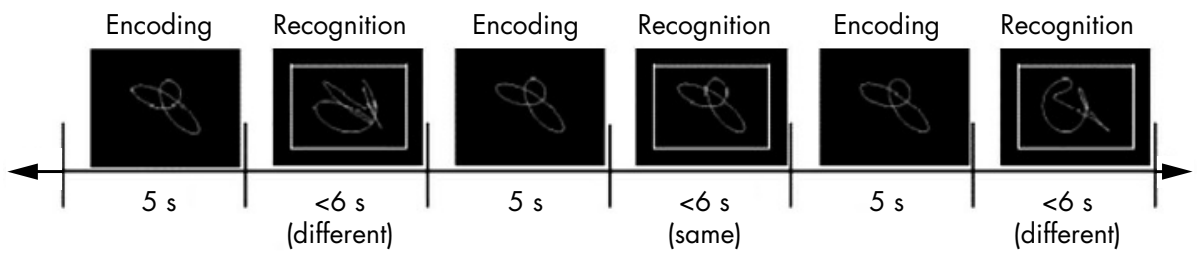

Titrated demand condition

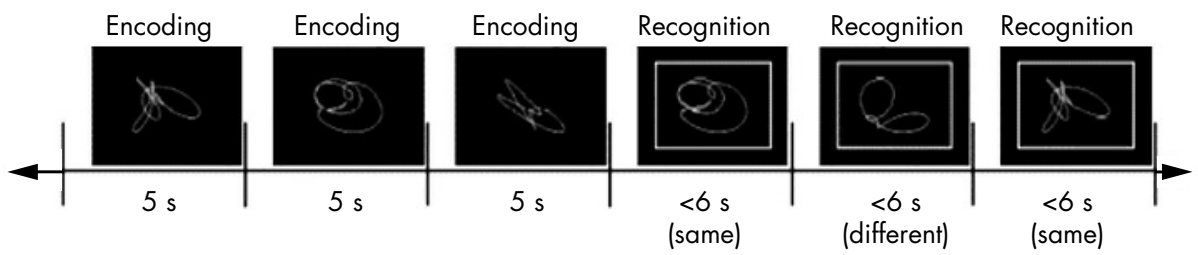

Figure 1 Non-verbal memory task. During the simple demand (SD) condition a single shape (the same each time) was presented during the encoding phase, followed by one shape during the recognition phase (either the same as the encoding phase shape or a non-familiar foil). The titrated demand (TD) condition involved a serial presentation of a sequence of shapes during the encoding phase; the length of the sequence was determined in a training session on the day preceding the PET scan during which shape list size was adjusted in a staircase manner such that recognition accuracy of about 75\% for each individual subject was attained; the recognition phase of the TD condition involved presentation of shapes studied during the preceding encoding phase intermixed with non-familiar foils. For presentation purposes, a list length of three shapes during the TD condition is illustrated here. During the recognition phases of both SD and TD conditions subjects were instructed to make a "new" or "old" response for each probe item (distinguished from encoding items by a rectangular frame) by pressing one of two microswitches. "New" and "old" test probes each occurred with a frequency of 50\%. Test probes were pseudorandomised so that no more than four consecutive trials required the same response. Each shape was used only once for each subject.

MNI coordinates were converted to standard Talairach brain atlas coordinates. A search for the closest grey matter Brodmann areas was carried out using the "Talairach Demon" database server http://biad02.uthscsa.edu/RIC WWW.data/Components/talairach/talairachdaemon.html.

\section{RESULTS}

Age, education, and neuropsychological performance did not differ among the groups (table 1). There was no association between APOE genotype and ethnicity: $\epsilon 4$ carriers, three white and one African American; non- $€ 4$ carriers, 11 white, one African American, one Hispanic, and three other ethnicities $\left(\chi^{2}=2.1, \mathrm{p}=0.54\right)$. All $\in 4$ carriers and seven non- $€ 4$ carriers were male $(p=0.09$, Fisher's exact test). Neither shape recognition accuracy nor shape list length were related to the APOE genotype.
In comparison with the subjects without the $€ 4$ allele, $\in 4$ carriers showed significant deactivation in right superior temporal and left fusiform gyri (table 2, fig 2, fig 3). E4 carriers showed significantly higher activation in the left middle temporal and right transverse temporal gyri (table 2 , fig 2, fig 3). The results were also significant in the nonparametric test. Because of the slight (although nonsignificant) imbalance in sex distribution, we conducted supplementary analyses including sex as a covariate; the results were essentially unchanged.

For all 20 subjects combined there were significant activations ( $t$ values ranging from 4.51 to 8.11 ) during the TD as compared to the SD condition in bilateral occipitalparietal areas (Brodmann areas (BA) 18, 19; middle occipital gyri, cuneus and fusiform gyri), right inferior parietal lobule (BA 40), right inferior (BA 9), and left superior (BA 10) and middle frontal (BA 9) gyri. Significant deactivations ( $t$ values

Table 1 Demographic and neuropsychological performance information by APOE group

\begin{tabular}{|c|c|c|c|c|c|}
\hline & \multicolumn{2}{|c|}{ No $€ 4$ allele $\epsilon 2 / \epsilon 3(n=2), \epsilon 3 / \epsilon 3(n=14)$} & \multicolumn{2}{|c|}{ Presence of $\epsilon 4$ allele $\epsilon 3 / \epsilon 4 \quad(n=4)$} & \multirow[b]{2}{*}{ p Value } \\
\hline & Mean (SD) & Range & Mean (SD) & Range & \\
\hline Age (years) & $23.1(2.25)$ & 19 to 28 & $25.3(1.23)$ & 24 to 27 & 0.09 \\
\hline Education (years) & $16.8(1.83)$ & 13 to 20 & $17.0(2.45)$ & 14 to 19 & 0.87 \\
\hline $\mathrm{mMMS}$ & $55.6(1.03)$ & 53 to 57 & $56.0(1.41)$ & 54 to 57 & 0.55 \\
\hline NART IQ & $120.8(3.19)$ & 115.2 to 126.6 & $122.3(4.64)$ & 115.8 to 125.7 & 0.47 \\
\hline WAIS-R vocabulary & $13.6(1.37)$ & 10 to 15 & $12.5(2.38)$ & 9 to 14 & 0.25 \\
\hline SRT total recall & $59.1(6.31)$ & 49 to 69 & $55.0(8.83)$ & 43 to 63 & 0.29 \\
\hline SRT delayed recall & $10.4(1.50)$ & 7 to 12 & $9.5(1.73)$ & 8 to 11 & 0.29 \\
\hline WAIS-R digit symbol & $12.5(2.94)$ & 6 to 19 & $10.8(4.27)$ & 5 to 14 & 0.34 \\
\hline Recognition accuracy (\% of shapes) & $0.81(0.11)$ & 0.55 to 0.95 & $0.79(0.13)$ & 0.61 to 0.92 & 0.77 \\
\hline TD shape list length & $14.9(6.69)$ & 5 to 35 & $14.8(4.92)$ & 10 to 19 & 0.96 \\
\hline
\end{tabular}

Student's $t$ test was used for $p$ value calculations

mMMS, modified mini-mental state examination; NART IQ, Nelson adult reading test IQ; SRT, selective reminding test; TD, titrated demand; WAIS-R, Wechsler adult intelligence scale-revised. 
Table 2 Areas where significant associations between brain activation differences (TD - SD) and APOE (presence versus absence of $\epsilon 4)$ were detected ( $p<0.05$ Bonferroni corrected) in the SPM analyses

\begin{tabular}{|c|c|c|c|c|c|c|}
\hline & \multicolumn{3}{|c|}{ Talairach Coordinates } & \multirow{2}{*}{$\begin{array}{l}t \text { Values } \\
(\mathrm{df}=18)\end{array}$} & \multirow{2}{*}{$\begin{array}{l}\text { Cluster size } \\
\text { (voxels) }\end{array}$} & \multirow[b]{2}{*}{ Location (Brodmann's area) } \\
\hline & $\mathbf{x}$ & $y$ & $\mathbf{z}$ & & & \\
\hline $\begin{array}{l}\text { Areas where } \epsilon 4 \text { carriers } \\
\text { showed lower rCBF activation }\end{array}$ & $\begin{array}{r}53 \\
-53\end{array}$ & $\begin{array}{r}10 \\
-36\end{array}$ & $\begin{array}{l}-10 \\
-27\end{array}$ & $\begin{array}{l}5.5 \\
5.3\end{array}$ & $\begin{array}{l}24 \\
20\end{array}$ & $\begin{array}{l}\text { Right superior temporal gyrus (38) } \\
\text { Left inferior temporal-fusiform gyrus (20) }\end{array}$ \\
\hline $\begin{array}{l}\text { Areas where } \epsilon 4 \text { carriers } \\
\text { showed higher } \mathrm{rCBF} \text { activation }\end{array}$ & $\begin{array}{r}-42 \\
36\end{array}$ & $\begin{array}{l}-56 \\
-31\end{array}$ & $\begin{array}{r}-4 \\
11\end{array}$ & $\begin{array}{l}5.6 \\
5.3\end{array}$ & $\begin{array}{l}12 \\
18\end{array}$ & $\begin{array}{l}\text { Left middle temporal gyrus (37) } \\
\text { Right transverse temporal gyrus (41) }\end{array}$ \\
\hline
\end{tabular}

4.69 to 14.15 ) during the TD (as compared to SD) condition were noted in the anterior cingulate area (BA 32), bilateral temporal areas (BA 21, 22, 39; superior and middle temporal gyri), bilateral parietal areas (BA 40, 39; inferior parietal lobule and angular gyrus), bilateral insula (BA 13), bilateral frontal (BA 11, 9, 47; medial, middle and inferior frontal gyri), left fusiform, and lingual gyri (BA 20, 18), and putamen. There was no overlap between the above areas and regions where the cognitive task condition $\times$ APOE interaction was significant.

Areas where $\in 4$ allele carriers had significantly lower rCBF activation

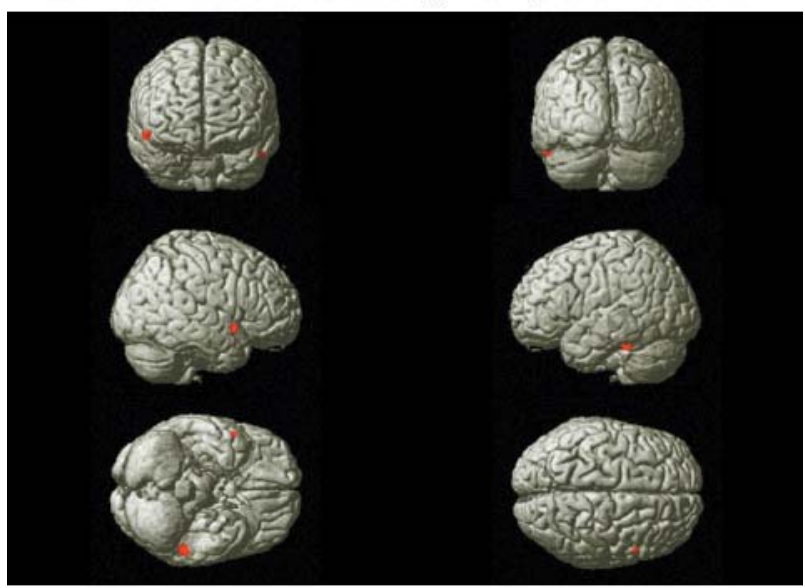

Areas where $\epsilon 4$ allele carriers had significantly higher rCBF activation

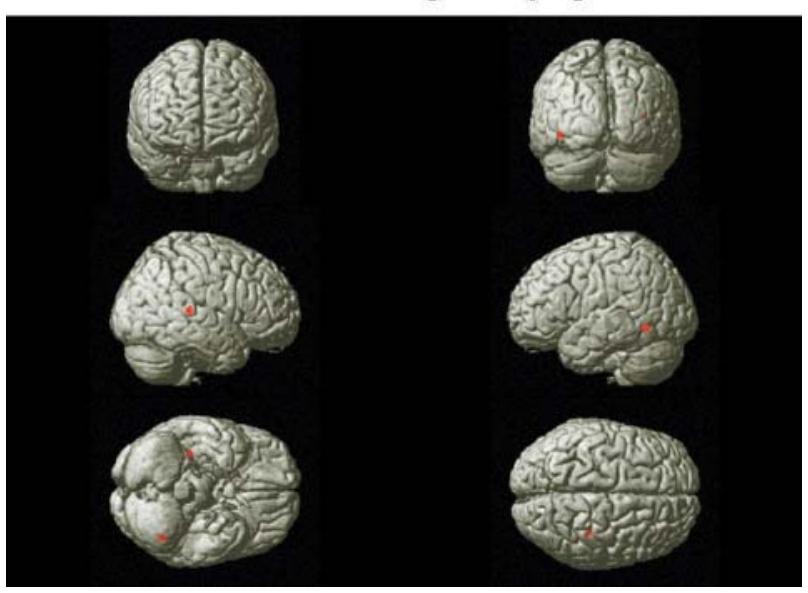

Figure 2 Three dimensional brain rendering representation of statistical parametric mapping depicting areas of significant mean brain activation ( $p<0.05$, Bonferroni corrected): relative cerebral blood flow differences (titrated demand minus simple demand) between subjects with and without an $\in 4$ allele.

\section{DISCUSSION}

We observed differences in brain activation in healthy young subjects with different APOE genotypes. College age healthy $\epsilon 4$ carriers, although cognitively indistinguishable from non$\epsilon 4$ carriers, showed areas of significantly different activation (either higher or lower) during the non-verbal memory task. These differences were not a reflection of task difficulty (which was equated in our experimental design) but indicate memory related altered cerebral physiology in young subjects with the $\epsilon 4$ allele.

There are known early biochemical changes in neuronal processes and synapses, long before structural Alzheimer's disease pathology is detected. ${ }^{19}$ It is conceivable that these changes may be manifested in rCBF activation studies. It is also known that symptoms of Alzheimer's disease are preceded by a period of unknown duration during which neuropathological alterations accumulate in the brain without associated memory loss or other detectable cognitive change. In a neuropathological study of 105 necropsy cases who showed no signs of dementia, abnormally high brain $\beta$ amyloid levels were reported for $€ 4$ carriers as young as 40 years of age. ${ }^{20}$ That study concluded that the $\epsilon 4$ allele predisposes the carriers to begin accumulating $\beta$ amyloid earlier in life than non-carriers. In another study, the $\epsilon 4$ allele was associated with the presence of neurofibrillary tangle changes in 44 necropsy cases of young subjects (mean age 38 years, range 22 to 46$).{ }^{21}$ The usual pattern seen in PET imaging of Alzheimer patients includes parietal, temporal, cingulate, and sometimes prefrontal hypometabolism or hypoperfusion. ${ }^{22-26}$ The $\epsilon 4$ related differences in activation were localised in the temporal areas in our study. Thus incipient Alzheimer's disease type pathology may be present in $€ 4$ carriers even from a very young age, while the brain preserves enough redundancy/efficiency to avoid failure in clinical cognitive performance.

However, several considerations make this possibility less plausible. The fact that the APOE related differences in activation are detected at such a young age (four to five decades before the possible onset of dementia) weakens the hypothesised link with Alzheimer's disease. The spatial extend of differences in activation between $\epsilon 4$ carriers and non-carriers is small and both increased and decreased activation was noted for $\epsilon 4$ carriers. Most resting flow or metabolism studies have reported decreased signal at parietal association areas but we did not detect differences in this location. The $\epsilon 4$ allele's presence is not equivalent to early Alzheimer's disease, and a significant proportion of $\epsilon 4$ heterozygotes will never develop Alzheimer's disease. It has been proposed that $\epsilon 4$ facilitates rather than causes the disorder ${ }^{27}$ and $\epsilon 4$ has been implicated in impaired brain repair mechanisms which may place subjects at risk for either Alzheimer's disease or other brain diseases. Important direct effects of the $\epsilon 4$ allele on the nervous system include impaired neuroregeneration within the dentate gyrus ${ }^{28}$ and increased vulnerability to exogenous neurotoxins. ${ }^{29}$ The 

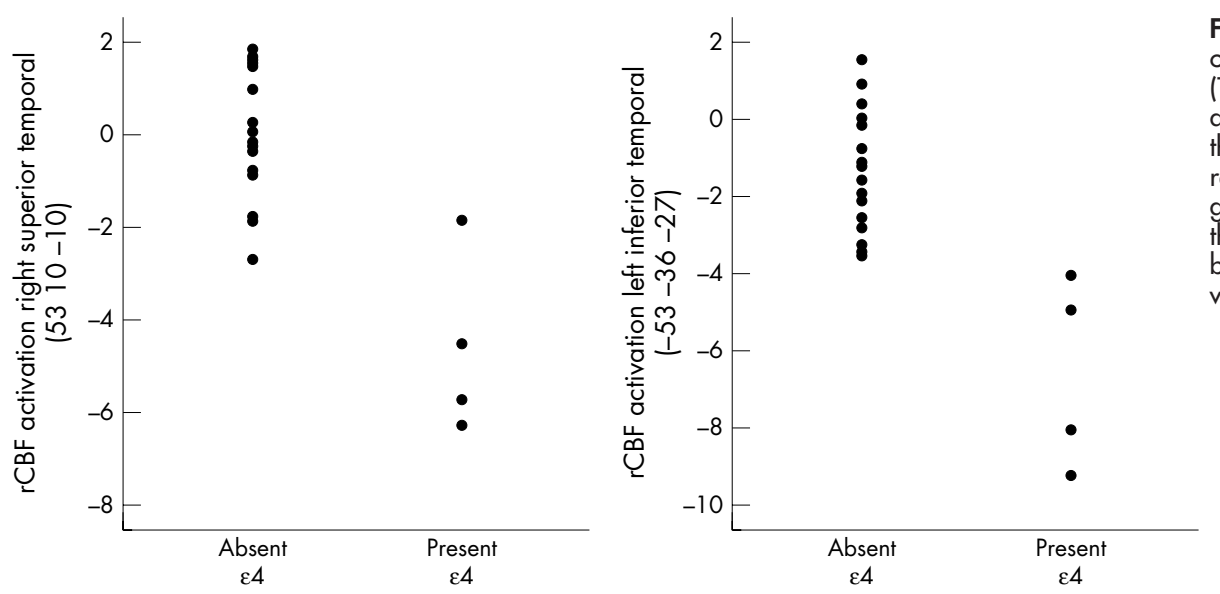

Figure 3 Graphs plotting relative cerebral blood flow (rCBF) activation (TD-SD) for the two groups. Note the almost clear rCBF separation between the $\epsilon 4$ carriers and non-carriers in these regions. In the right transverse temporal gyrus there is a visual impression of three (rather than four) $\in 4$ carriers because of close proximity of $\mathrm{rCBF}$ values.
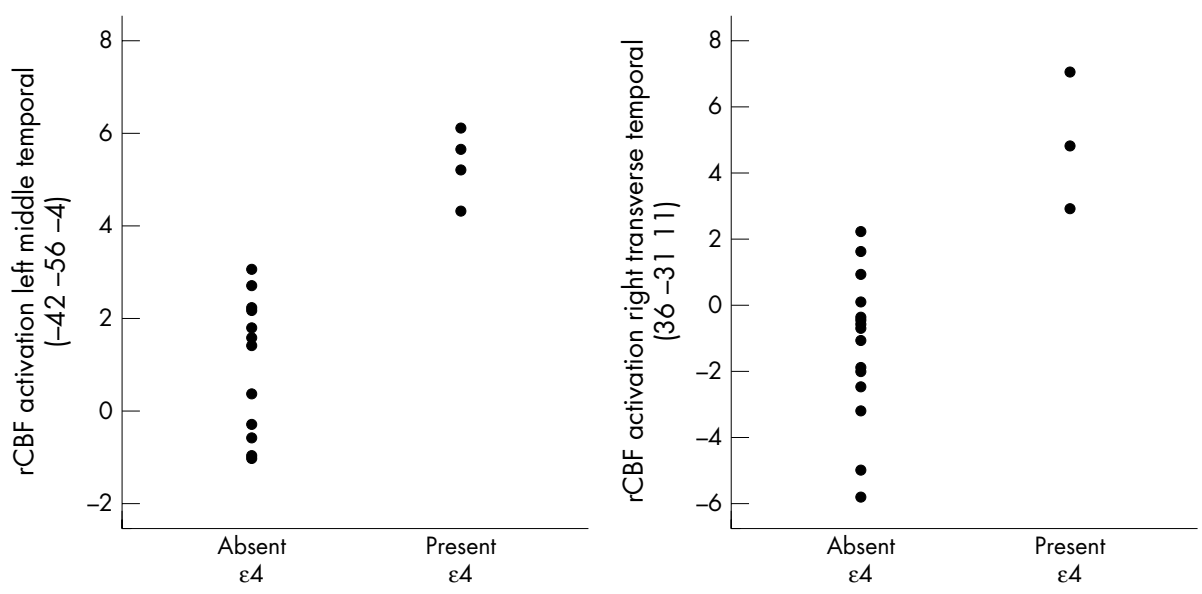

observed activation pattern of $\epsilon 4$ carriers in their twenties may be the early signature of an APOE dependent alteration in brain physiology which could result in greater vulnerability to environmental effects (such as traumatic brain injury or other insult) later in life. It is also conceivable that differences in cerebral activation may not be strictly APOE related but may be present as a function of many neurally expressed genes other than APOE. Thus the detected activation differences may reflect an APOE dependent physiological heterogeneity which may not necessarily lead to disease.

In PET studies during rest, the inheritance of the $\epsilon 4$ allele has been associated with decreased parietal, ${ }^{30}{ }^{31}$ temporal, cingulate, and prefrontal ${ }^{31}$ metabolism (as with the PET pattern seen in Alzheimer's disease) in middle aged adults with a family history of Alzheimer's disease. Resting imaging studies in young people have revealed decreased temporal, ${ }^{16}$ parietal, cingulate, and prefrontal ${ }^{17}$ metabolism or perfusion, but also increased insula, supramarginal, and superior occipital gyral ${ }^{16}$ perfusion for the $\epsilon 4$ carriers. All detected differences in activation in our study were localised to the temporal regions where alterations in resting metabolism or flow have been noted for both middle aged and young $\epsilon 4$ carriers.

Only four previous studies have investigated APOE related brain activation, with mixed results. One study reported increases in the extent and intensity of activation during learning and recall periods in (among other regions) temporal areas in middle aged and elderly $\epsilon 4$ allele carriers, ${ }^{12}$ which was interpreted as an attempt to ameliorate the effects of reduced function in the networks that normally operate during the task. We also noted increased activation for the $\epsilon 4$ carriers in temporal areas. However, unlike the above study, we also found areas of decreased activation for the $\epsilon 4$ carriers. Decreased activation for $\epsilon 4$ carriers has been reported in two other studies-one of middle aged women with at least one first degree relative with Alzheimer's disease during a letter fluency task ${ }^{1432}$ and one of cognitively intact elderly people while performing the same non-verbal memory task. ${ }^{11}$ As compared with the above two studies, the locations of decreased activation in the present study were less extended and spatially close (but not identical). Previously reported increased activations for $\epsilon 4$ carriers in prefrontal ${ }^{12}$ or parietal areas ${ }^{12}{ }^{14}$ were not noted in our study. In contrast to previous reports, no APOE related differences were detected in another study of elderly subjects using a verbal working memory task at various levels of task difficulty. ${ }^{13}$ The investigators concluded that the effect of APOE is specific to episodic encoding and not related to general task difficulty. We detected APOE related differences in our study despite having titrated shape list length, which supports the above notion that the association between APOE genotype and cerebral activation does not seem to be equated to task difficulty.

Many factors may account for discrepancies in the findings between the present and previous activation studies: different imaging modes (all but one previous studies used BOLDfMRI, where the signal is the result of a complex interaction between changes not only of blood flow but also of blood volume and oxygen consumption accompanying neural activity), differences in power (more spatially restricted activations or deactivations in the current study may be the result of fewer subjects or different relative proportions of homozygotes to heterozygotes), and differences among the 
populations studied (demographics, ethnicity, neuropsychological performance, family history of Alzheimer's disease, recruitment methods, and so on). In particular, all previous studies have been done in middle aged or elderly people. Also the present study equated for task difficulty, an experimental design not used in previous studies (activation differences have to be considered under the factor of increased task complexity, because activations may reflect modulation of the same network in response to differential difficulty rather than APOE related recruitment). Finally, cognitive activation tasks have differed across studies. Previous cognitive tasks have included a verbal fluency test, ${ }^{14}$ a covert object naming test, ${ }^{32}$ a paired associates verbal recall test, ${ }^{12}$ and a digit span working memory task. ${ }^{13}$ The present task is likely to make demands on episodic memory encoding, storage, and retrieval but also frontoparietal networks underlying shifts of spatial attention. ${ }^{33-35}$

This is the first report indicating that $€ 4$ allele carriers have alterations in brain activation even at very young age when behavioural, cognitive, or clinical evidence of disease is absent. Although the results were similar in a sex adjusted model, the absence of sex matching could be a confounding factor. Because of the relatively small number of participants (in particular for the $\epsilon 4$ group) the results can only be viewed as preliminary and they should be confirmed in a larger study. However, the significant associations noted indicate that there were effects strong enough to be demonstrable even with our conservative approach to type I error.

\section{ACKNOWLEDGEMENTS}

This research was supported by federal grants AG 14671 and RR 00645.

\section{Authors' affiliations \\ N Scarmeas, C G Habeck, J Hilton, J Flynn, A Park, Cognitive} Neuroscience Division of the Taub Institute for Research in Alzheimer's Disease and the Aging Brain, Columbia University Medical Center, New York, USA

Y Stern, Departments of Neurology, Columbia University Medical Center, New York, USA

K E Anderson, Department of Psychiatry, University of Maryland

Competing interests: none declared

Correspondence to: Dr Nikolaos Scarmeas, Columbia University Medical Center, 622 West 168th street, PH 19th floor, New York, NY 10032, USA; ns257@columbia.edu

Received 8 September 2004

In revised form 26 January 2005

Accepted 3 February 2005

\section{REFERENCES}

1 Srinivasan SR, Ehnholm C, Elkasabany A, et al. Apolipoprotein E polymorphism modulates the association between obesity and dyslipidemias during young adulthood: the Bogalusa Heart Study. Metabolism 2001;50:696-702.

2 Katsuya T, Baba S, Ishikawa K, et al. Epsilon 4 allele of apolipoprotein E gene associates with lower blood pressure in young Japanese subjects: the Suita Study. J Hypertens 2002;20:2017-21.

3 Hixson JE. Apolipoprotein E polymorphisms affect atherosclerosis in young males. Pathobiological Determinants of Atherosclerosis in Youth (PDAY) Research Group. Arterioscler Thromb 1991;11:1237-44.

4 van Bockxmeer FM, Mamotte CD. Apolipoprotein epsilon 4 homozygosity in young men with coronary heart disease. Lancet 1992;340:879-80.

5 Brscic $\mathbf{E}$, Bergerone S, Gagnor A, et al. Acute myocardial infarction in young adults: prognostic role of angiotensin-converting enzyme, angiotensin II type I receptor, apolipoprotein $E$, endothelial constitutive nitric oxide synthase, and glycoprotein Illa genetic polymorphisms at medium-term follow-up. Am Heart J 2000;139:979-84.
6 Ferguson SC, Deary IJ, Evans JC, et al. Apolipoprotein-e influences aspects of intellectual ability in type 1 diabetes. Diabetes 2003;52:145-8.

7 Rask-Nissila L, Jokinen E, Viikari J, et al. Impact of dietary intervention, sex, and apolipoprotein $\mathrm{E}$ phenotype on tracking of serum lipids and apolipoproteins in 1- to 5-year-old children: the Special Turku Coronary Risk Factor Intervention Project (STRIP). Arterioscler Thromb Vasc Biol 2002;22:492-8.

8 Tammi A, Ronnemaa T, Rask-Nissila L, et al. Apolipoprotein E phenotype regulates cholesterol absorption in healthy 13-month-old children - the STRIP Study. Pediatr Res 2001:50:688-91.

9 Zhou Y, Elkins PD, Howell LA, et al. Apolipoprotein-E deficiency results in an altered stress responsiveness in addition to an impaired spatial memory in young mice. Brain Res 1998;788:151-9.

10 Valastro B, Ghribi O, Poirier J, et al. AMPA receptor regulation and LTP in the hippocampus of young and aged apolipoprotein E-deficient mice. Neurobiol Aging $2001 ; 22: 9-15$.

11 Scarmeas N, Habeck C, Anderson KE, et al. Altered PET functional brain responses in cognitively intact elderly persons at risk for Alzheimer disease (carriers of the \{epsilon\}4 Allele). Am J Geriatr Psychiatry 2004; 12:596-605.

12 Bookheimer SY, Strojwas MH, Cohen MS, et al. Patterns of brain activation in people at risk for Alzheimer's disease. N Engl J Med 2000;343:450-6.

13 Burggren AC, Small GW, Sabb FW, et al. Specificity of brain activation patterns in people at genetic risk for Alzheimer disease. Am J Geriatr Psychiatry 2002;10:44-51

14 Smith CD, Andersen AH, Kryscio RJ, et al. Women at risk for AD show increased parietal activation during a fluency task. Neurology 2002; 58: 1197-202.

15 Scarmeas N, Anderson KE, Hilton J, et al. APOE-dependent PET patterns of brain activation in Alzheimer disease. Neurology 2004;63:913-15.

16 Scarmeas N, Habeck CG, Stern Y, et al. APOE genotype and cerebral blood flow in healthy young individuals. JAMA 2003;290:1581-2.

17 Reiman EM, Chen K, Alexander GE, et al. Functional brain abnormalities in young adults at genetic risk for late-onset Alzheimer's dementia. Proc Natl Acad Sci USA 2004;101:284-9.

18 Scarmeas N, Zarahn E, Anderson KE, et al. Cognitive reserve modulates functional brain responses during memory tasks: a PET study in healthy young and elderly subjects. Neuroimage 2003;19:1215-27.

19 Selkoe DJ. Alzheimer's disease is a synaptic failure. Science 2002;298:789-91

20 Morishima-Kawashima M, Oshima N, Ogata H, et al. Effect of apolipoprotein E allele epsilon4 on the initial phase of amyloid beta-protein accumulation in the human brain. Am J Pathol 2000;157:2093-9.

21 Ghebremedhin E, Schultz C, Braak E, et al. High frequency of apolipoprotein $\mathrm{E}$ epsilon4 allele in young individuals with very mild Alzheimer's diseaserelated neurofibrillary changes. Exp Neurol 1998;153:152-5

22 Silverman DH, Small GW, Chang CY, et al. Positron emission tomography in evaluation of dementia: Regional brain metabolism and long-term outcome. JAMA 2001;286:2120-7.

23 Hoffman JM, Welsh-Bohmer KA, Hanson M, et al. FDG PET imaging in patients with pathologically verified dementia. J Nucl Med 2000;41:1920-8.

24 DeCarli C, Atack, JR, Ball MJ, et al. Post-mortem regional neurofibrillary tangle densities but not senile plaque densities are related to regional cerebral metabolic rates for glucose during life in Alzheimer's disease patients. Neurodegeneration 1992;1:113-21.

25 Minoshima S, Frey KA, Koeppe RA, et al. A diagnostic approach in Alzheimer's disease using three-dimensional stereotactic surface projections of fluorine-18-FDG PET. J Nucl Med 1995:36:1238-48.

26 Kennedy AM, Frackowiak RS, Newman SK, et al. Deficits in cerebral glucose metabolism demonstrated by positron emission tomography in individuals at risk of familial Alzheimer's disease. Neurosci Lett 1995;186:17-20.

27 Meyer MR, Tschanz JT, Norton MC, et al. APOE genotype predicts when - not whether - one is predisposed to develop Alzheimer disease. Nat Genet $1998 ; 19: 321-2$.

28 Teter B, Xu PT, Gilbert JR, et al. Human apolipoprotein E isoform-specific differences in neuronal sprouting in organotypic hippocampal culture. J Neurochem 1999:73:2613-16.

29 Buttini M, Orth M, Bellosta S, et al. Expression of human apolipoprotein E3 or E4 in the brains of Apoe-/- mice: isoform-specific effects on neurodegeneration. J Neurosci 1999; 19:4867-80.

30 Small GW, Mazziotta JC, Collins MT, et al. Apolipoprotein E type 4 allele and cerebral glucose metabolism in relatives at risk for familial Alzheimer disease. JAMA 1995;273:942-7.

31 Reiman EM, Caselli RJ, Yun LS, et al. Preclinical evidence of Alzheimer's disease in persons homozygous for the epsilon 4 allele for apolipoprotein $\mathrm{E}$. N Engl J Med 1996;334:752-8.

32 Smith CD, Andersen AH, Kryscio RJ, et al. Altered brain activation in cognitively intact individuals at high risk for Alzheimer's disease. Neurology 1999;53:1391-6

33 Anderson KE, Lynch K, Zarahn E, et al. PET O-15 study of impairment of nonverbal recognition with normal aging. J Neuropsychiatry Clin Neurosci, (in press).

34 Stern Y, Zarahn E, Hilton HJ, et al. Exploring the neural basis of cognitive reserve. J Clin Exp Neuropsychol 2003;25:691-701.

35 Habeck C, Hilton HJ, Zarahn E, et al. Relation of cognitive reserve and task performance to expression of regional covariance networks in an eventrelated fMRI study of nonverbal memory. Neuroimage 2003;20:1723-33. 